\title{
Self-adjusted multi-sensor information fusion electric energy measuring based on neural networks
}

\author{
ZhuFeng $\mathrm{Li}^{1}$,Yang Li ${ }^{2}$ \\ ${ }^{1}$ College of Application Physics and material,WuYi Univ. 529020 jiangmeng Guangdong China \\ ${ }^{2}$ Student, College of Biological Sciences, State university of Minnesota . 55401 st.paul Minnesota United States
}

\begin{abstract}
In this article, self-adjusted Multi-sensor Information Fusion measuring method of electric energy based on neural networks has been thoroughly given. This paper studies the method of automatic error correction of electric power measurement also . The effective learning algorithm of the neural network based on gradient algorithm and Newton algorithm is combined with the LEA discriminant method.The results show that the method can improve the learning efficiency. The hardware model of adaptive real-time fast power measurement is constructed by using DSP device. The experimental results show that the adaptive power measurement model is better than the traditional power meter.
\end{abstract}

KeyWords : neural networks、 power energy measuring、self-adjusted 、DSP

\section{Introduction}

High precision measurement of electric energy in complex field environment is an urgent problem that has not been solved. Such as temperature $-30-+40{ }^{\circ} \mathrm{C}$, humidity of $40 \%-80 \%$, frequency, voltage, current, power factor, external magnetic field, waveform distortion, voltage line loss and so on. These factors seriously affect the accuracy of electrical energy measurement.The economic loss caused by inaccurate measurement of electric energy can only be artificially estimated, which is unscientific and unfair. The failure of the metering device can not be found in time and non manual. Fault can only be detected when waiting for cycle check, Resulting in time delays and economic losses. How to improve the measurement error of the electric power measurement device in a complex and changeable environment,It is of great significance to realize the real-time intelligent correction, to improve the accuracy of the electric energy meter in the complex and changeable environment, and to carry out the intelligent diagnosis of the fault of the metering device.The multi sensor information fusion based on the optimized neural network can effectively correct the influence of the environment on the electric energy measurement, and can realize the real-time, dynamic and high-precision intelligent electric energy measurement[1].Optimization of neural network is to determine a reasonable neural network structure, the optimal neural network weight learning algorithm.According to the actual situation, the establishment of a reasonable data sample library of environmental factors and power relations, measuring device status information and fault classification samples, It is of great academic and practical value to realize the accurate measurement and fault diagnosis of electric field measurement device[2,3,4].

\section{In the complex scene,basic principle of electric energy measurement and}

\section{fault diagnosis by the optimization of multi sensor information fusion based on neural network method}

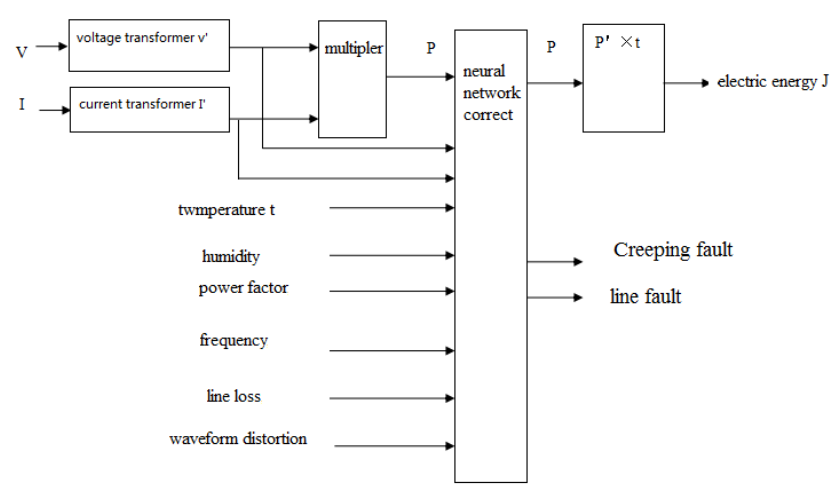

Fig.1 Electric energy measurement and fault diagnosis system scheme

Based on the optimized neural network and multi sensor information fusion, electric energy measurement and fault diagnosis system scheme is shown in figure 1.The voltage transformer transforms the high voltage into a smaller voltage V'. The current transformer transforms the high current into a small current I'. The electrical power $\mathrm{P}$ can be obtained by multiplying the voltage $\mathrm{V}^{\prime}$ with the current I'. According to the measurement results of multi sensors for field environment (temperature, humidity, voltage, frequency, current, power factor, magnetic field, waveform distortion, voltage line loss, etc.), real time and error corrected power P' can be obtained by neural network computing. P 'multiplied by time $t$ is the result of electrical energy $\mathrm{E}$.

The neural network needs to be optimized and trained before use.The sample database that maps the actual data relationship needs to be collected to built. Firstly, the fitness function of the network structure is established. Then, based on the genetic algorithm to optimize the weights of neural network, the neural network structure is optimized, To search for a reasonable neural network structure for the application of 
multi sensor information fusion in complex field environment. According to the state information (power, current, voltage, power factor, line loss, etc.),real time fault diagnosis of electric energy measurement device can be completed by neural network.Creeping, large line loss and disconnection are part of the fault state information .Neural network needs to complete information fusion learning,clustering learning,finally realize the diagnosis function[5,6].

\section{Optimization methods of neural network structure}

According to the complexity of the specific problems, the appropriate neural network structure can be selected, that is, to ensure the network of data induction, but also save resources and time.The number of nodes in the input layer is determined by the physical quantity of the detection. The number of nodes in the output layer is determined according to the number of outputs.It is difficult to determine the number of hidden nodes.Considering the network capacity and function approximation, the number of hidden units should be chosen more.However, considering the generalization ability of the network, the number of hidden units should not be too much.So the number of hidden units should have the best value.The initial number of hidden units should be given first, and then the correlation pruning algorithm is used to reduce the number of hidden nodes until the number of hidden nodes is determined.Finally, the number of nodes of hidden units with less and reasonable number is determined.The initial number of hidden units is determined by subtractive clustering method[7].

The method of determining the initial number of hidden units (subtractive clustering method).

Set $\mathrm{m}$ dimensional space $\mathrm{n}$ data points $\left(\mathrm{X}_{1}, \mathrm{X}_{2}, \ldots, \mathrm{X}_{\mathrm{n}}\right)$, subtractive clustering process is as below. first, for each point $X_{i}$ of the data set, the density index is calculated.The highest density data $\mathrm{X}_{\mathrm{ci}}$ is the first cluster center.

$$
D_{i}=\sum_{j=1}^{n} \exp \left[-\left\|x_{i}-x_{j}\right\|^{2} /\left(0.5 r_{a}\right)^{2}\right] \ldots \ldots . .(1)
$$

$\mathrm{X}_{\mathrm{ck}}$ is selected as the cluster center of $\mathrm{K}$, and the corresponding density index is $\mathrm{D}_{\mathrm{ck}}$, and the density function of each data point is modified by the following formula

$$
D_{i}^{c_{i}}=D_{i}-D_{c k} \exp \left[-\left\|x_{i}-x_{c k}\right\|^{2} /\left(0.5 r_{b}\right)^{2}\right]
$$

The $\mathrm{X}_{\mathrm{c}(\mathrm{k}+1)}$, which has the highest density index, is a new clustering center.

If the condition $\mathrm{D}_{\mathrm{c}(\mathrm{k}+1)} / \mathrm{D}_{1}<\delta$ is not established, the application formula (2) can be used to select the next cluster center.If it is set up, the clustering results and the number of clusters (i.e. the initial number of hidden layers) are given.the parameters $\delta<1$ is based on empirical parameters. This parameter determines the final number of initialization cluster centers, the smaller the $\delta$, the more the number of clusters.

$$
r_{a}=r_{b}=\frac{1}{2} \min _{k}\left\{\max \left\{\left\|x_{i}-x_{k}\right\|\right\}\right\} \cdots \cdots \cdots . . .(3)
$$

The neural network consists of three layers: input layer, hidden layer and output layer.Neural network has a network structure with parallel and distributed information processing, With learning, induction, extraction and other functions. The input layer receives the input letter from the system.According to the input information, the hidden layer can be used for internal learning and processing, which is connected with the input layer and the output layer through the right.The output layer obtains the desired result according to the input and adjustment weight coefficients.Input layer input information is power, temperature, humidity, frequency, voltage, current, magnetic field strength etc.The output layer uses the actual power output value $\mathrm{Y} 1$, and the other outputs are used for function development.The actual power output value Y1 is multiplied with time and accumulated into the measured power energy.Power expectation $\mathrm{B}$ is provided by standard power meter. The accuracy of the standard power meter is relatively small, and it is only used in the neural network learning stage to determine the connection weights of the neural network. The standard power meter is not used for the actual measurement of electric energy. The actual power output Y1 should be close to the corresponding power expectation value $\mathrm{B}$ under various conditions calculated by neural network .According to the input value of power measurement $\mathrm{X} 1$ and the change of the environment quantity, by the neural network calculating, and the correction value adjusted selfadaption, high precision power can be obtained.As a result, it can realize the adaptive adjustment of the error in the power measurement. finally the adaptive error correction of the electric energy measurement is realized.[7,8]

\section{4 learning methods}

BP neural network model shown in figure 2.The learning process of the network algorithm consists of two parts: forward and backward propagation. In the process of forward propagation, the input information is transmitted from the input layer to the output layer by layer by layer. The state of each neuron affects only the state of the next neuron. If the desired output is not obtained in the output layer, then it is transmitted back, and the error signal is returned along the original path, and the error signal is minimized by modifying the weights of the neurons in each layer. $[9,10,11]$

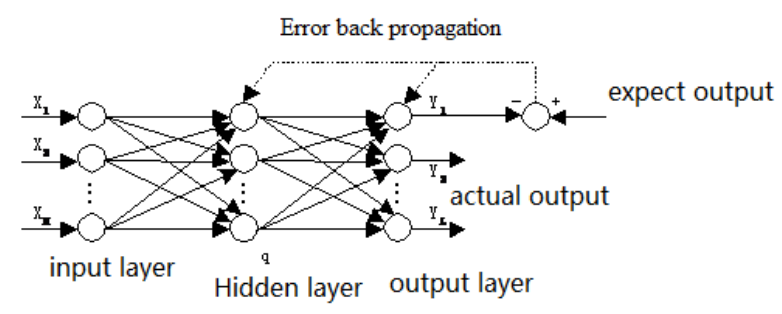

Fig . 2 Bp neural network model

Set the input vector $\mathrm{X}_{\mathrm{I}}=\left(\mathrm{x}_{\mathrm{I} 1}, \mathrm{x}_{\mathrm{I} 2}, \ldots \mathrm{x}_{\mathrm{IM}}\right)$; output vector $\mathrm{B}_{\mathrm{I}}=$ $\left(b_{I 1}, b_{I 2}, \ldots B_{I L}\right)$; the actual output vector of the output layer unit $\mathrm{Y}_{\mathrm{I}}=\left(\mathrm{y}_{\mathrm{I} 1}, \mathrm{y}_{\mathrm{I} 2}, \ldots \mathrm{y}_{\mathrm{IL}}\right) ; \mathrm{I}=1,2, \ldots \mathrm{z}, \mathrm{z}$ is the number of samples.The connection weight of input layer to the hidden layer is $\{\mathrm{Uij}\}, \mathrm{i}=1,2, \ldots, \mathrm{m} ; \mathrm{j}=1,2, \ldots, \mathrm{q}$. The connection weight of the hidden layer to output layer is $\{\mathrm{Vjt}\}, \mathrm{j}=1,2, \ldots, \mathrm{q}$; $\mathrm{t}=1,2, \ldots$ L. The output threshold of each unit at the hidden layer is $\{\mathrm{Oj}\}, \mathrm{j}=1,2, \ldots, \mathrm{q}$. The response function of the hidden layer nodes is 
$f(x)=\left(1+e^{-x}\right)^{-1}$.The response function of the output notes is liner.

Formula (4) is the error energy function. That is, the output vector $\mathrm{B}_{\mathrm{I}}=\left(\mathrm{b}_{\mathrm{II}}, \mathrm{b}_{\mathrm{I}}, \ldots \mathrm{B}_{\mathrm{IL}}\right)$ subtract the actual output vector of the output layer unit $Y_{I}=\left(y_{11}, y_{12}, \ldots y_{I L}\right)$. Then ,the error squared is added.

$E=\frac{1}{2} \sum_{I=1}^{z} \sum_{t=1}^{L}\left[\sum_{j=1}^{q} v_{j t} f\left(\sum_{i=1}^{M} u_{i j} x_{I i}+\mathbf{o}_{j}\right)-b_{I t}\right]^{2}+\alpha e^{-\frac{1}{q+\beta n}}$

By adjusting the weights $\left\{\mathrm{u}_{\mathrm{ij}}\right\},\left\{\mathrm{v}_{\mathrm{jt}}\right\}$ and the number of hidden layer nodes $\mathrm{Q}, \mathrm{n}$ number of network connection weights, the error energy function $\mathrm{E}(\mathrm{w})$ can reach the minimum, to realize the optimization of the network selection. Then $\mathrm{E}$ is a function of $\mathrm{w}, E=E(w) \cdot[12,13,4]$

\subsection{Steepest gradient descent learning algorithm}

$\mathrm{W}$ is a vector, $\mathrm{E}$ is a multivariate function. Through the network learning, $w$ value can be determined, at this time the function $\mathrm{E}$ to achieve the minimum value.Gradient $\mathrm{G}(\mathrm{w})$ is vector. If $\mathrm{E}(\mathrm{w})$ produces the greatest change, the corresponding direction is $\mathrm{G}(\mathrm{w})$ direction. The mode is the maximum change rate of $\mathrm{E}(\mathrm{w})$ at $M$ point.Vector $G(w)$ is the gradient of the number field $\mathrm{E}(\mathrm{w})$ at the $\mathrm{w}$ point.grad $\left.\mathrm{E}\right|_{\mathrm{M}}=\mathrm{G}(\mathrm{W})=\nabla \mathrm{E}$.

$$
\operatorname{grad} E=\frac{\partial E}{\partial x} \dot{i}+\frac{\partial E}{\partial y} \vec{j}+\frac{\partial E}{\partial z} \vec{k}+\ldots \quad \nabla=\dot{i} \frac{\partial}{\partial x}+\vec{j} \frac{\partial}{\partial y}+\vec{k} \frac{\partial}{\partial z}+\ldots
$$

Hamiiton operator.

The gradient descent learning algorithm is shown as follows. The initial position $\mathrm{W}(\mathrm{k})$ is chosen randomly, and the moving direction and step size of $\mathrm{W}(\mathrm{k}+1)$ are determined according to the gradient of $\mathrm{W}(\mathrm{k})$ point, so that the error function $\mathrm{E}(\mathrm{W}(\mathrm{K}+1))$ is decreased rapidly than $\mathrm{E}(\mathrm{W})(\mathrm{K})$.Then $\mathrm{W}(\mathrm{k}+1)$ is used as the starting point and the steepest descent method is used repeat to determine the $\mathrm{W}(\mathrm{k}+2)$. Finally, the point $\mathrm{W}(\mathrm{k}+\mathrm{n})$, which former the smallest $\mathrm{E}(\mathrm{W})$ value, is found as the solution of the neural network weights.The iterative formula of steepest descent learning algorithm is formula (5).A (k) is a positive number, also known as the step size. At each iteration, a (k) is determined by the one dimensional linear search.The steepest gradient descent learning method decreased rapidly at the early stage of learning, and learning in the later there will be a severe vibration phenomenon that the learning speed is very slow, because two times the search direction of the adjacent gradient algorithm is orthogonal, the search process will appear "seesaw" phenomenon. Therefore, in the later stage of learning the Newton method is used.

$W(k+1)=W(k)-a(k) \nabla E(W(k))$

\section{Newton iteration method}

According to the BP neural network in Figure 2, the weights of BP neural network can satisfy the solution of nonlinear equations. The nonlinear equations are formula (6).

$$
\begin{aligned}
& \sum_{j=1}^{q} v_{j t} f\left(\sum_{i=1}^{M} u_{i j} x_{I j}{ }^{+} o_{j}\right)-b_{I t}=0=F(W) \ldots \ldots \ldots(6) \\
& \quad(\mathrm{t}=1,2, . . \mathrm{L}), \quad(\mathrm{I}=1,2, \ldots, \mathrm{Z}), .
\end{aligned}
$$

$W=\left(\boldsymbol{V}_{11}, \boldsymbol{v}_{12}, \cdots, \boldsymbol{v}_{q L}, \boldsymbol{U}_{11}, \boldsymbol{U}_{12}, \cdots, \boldsymbol{U}_{m q}, \boldsymbol{O}_{1}, \boldsymbol{O}_{2} \cdots, \boldsymbol{O}_{q}\right)$

$W$ is a vector of unknown weights. $\mathrm{F}=\left(\mathrm{f}_{11}, \mathrm{f}_{12}, \ldots \mathrm{f}_{\mathrm{ZL}}\right)^{\mathrm{T}} . \mathrm{F}$ is a nonlinear function. The nonlinear equations (6) have $\mathrm{q}$ $(\mathrm{M}+\mathrm{L}+1)$ unknown variables and $\mathrm{ZL}$ nonlinear equations. The nonlinear equations (6) is the objective function of the Newton iterative learning algorithm for solving the weights of neural networks.

$F^{\prime}(W(k))=\left[\frac{\partial f_{I t}}{\partial W_{i j}}\right]_{Z L \cdot q(M+L+1)}$

$F^{\prime}(W(k))$ is vector. The partial derivative of the function $\mathrm{F}$ is calculated separately for each variable.A matrix of partial derivatives is called the Jacobi matrix. Newton iterative method to calculate the weights of neural networks is formula (7).

$$
W(k+1)=W(k)-\left[F^{\prime}(W(k))\right]^{-1} F(W(K)) \ldots \ldots \ldots(7)
$$

$\left[F^{\prime}(W(k))\right]^{-1}$ is generalized inverse matrix of $F^{\prime}(\mathrm{W}(\mathrm{k}))$.In order to avoid the partial derivative calculation, the variation value can be used to replace the partial derivative in the Jacobi matrix, that is, using the approximate Newton method to complete the calculation. The applicable conditions of Newton method are as follows. The applicable conditions of Newton method are as follows. The choice of the initial point is sufficiently close to the extreme point ( $\mathrm{w}^{*}$ ), which is generated by the iterative computation to produce the sequence $\{\mathrm{W}(\mathrm{k})\}$, which is the solution of the nonlinear equations.If the initial point is far away from the pole, the Newton iteration method is likely to fail.As a result, the sequence obtained by iterative computation does not converge.Newton iterative method has the advantages of fast convergence speed and two order convergence rate.The gradient iteration method has only one order convergence rate. $[15,16,17]$

\subsection{The method of calculating the ratio of dislocation accumulation (CRDA method).}

When the gradient method fails, Newton method is used. CRDA method is a method to determine how to choose different methods to realize the combination of gradient method and Newton method.The CRDA method is proposed by the author of this thesis.For the gradient method, the error function in the initial stage of the neural network is decreased rapidly, and the selection of the initial point is not required.In the later period of study, there will be a violent vibration, which makes the learning speed slow. Newton iterative method has the advantage of fast convergence speed and two order convergence rate, but it is necessary to calculate the initial point near the poles.First, the gradient method is used to calculate the sequence point until it falls into the convergence domain of Newton method. Then, Newton method is used to realize the combination of gradient method and Newton method.An effective method is to give full play to the advantages of the two algorithms and make up for their deficiencies. The key question is when the gradient method is converted to Newton's method, which has a great influence on the learning effect.

In the case of the $\mathrm{K}$ iteration calculation, the sum of squared error function E (W (k-i)) is summed up. Only the fixed number of times (n) is calculated.A (k) represents the calculated results. $\mathrm{R}(\mathrm{K})$ is the ratio of the function $\mathrm{E}(\mathrm{W}(\mathrm{k}-1$ i)) with the function $\mathrm{E}(\mathrm{W}(\mathrm{k}-\mathrm{i}))$. That is, the location of the 
dislocation, to accumulate, the ratio, known as the dislocation plus CRDA method.

$$
\begin{aligned}
& A(k)=\sum_{i=0}^{n} E(W(k-i)) \\
& R(k)=\frac{A(k-1)}{A(k)}=\frac{\sum_{i=0}^{n} E(W(K-1-i))}{\sum_{i=0}^{n} E(W(k-i))}
\end{aligned}
$$

When $R(k) \rightarrow 1$, the gradient iteration method can be considered as a "seesaw" state, and the gradient algorithm is replaced by the Newton iteration method.

Formula(8) is the combination of gradient method and Newton method. When $\mathrm{R}(\mathrm{k})>1+\varepsilon, \varepsilon$ is a small positive number greater than $0, \lambda_{\mathrm{k}}=1$ is used and gradient iteration method is adopted. When $\mathrm{R}(\mathrm{k})=1+\varepsilon, \lambda_{\mathrm{k}}=0$ is selected, using Newton iterative method[29,30,31,32].The selection of epsilon will affect the iterative steps of the gradient iteration method. The and a I/O bus); 4M memory, with DMA controller and I/O processor, allowing flexible and high speed data transmission without overhead, the rate is $240 \mathrm{Mbytes} / \mathrm{s}$ can provide; and 16 32 bit processor interface; multi channel serial port with 40Mbit/s.In this paper, the DSP devices are used to receive the data of various environmental sensors and perform neural network calculation. The corrected power value is calculated, and then the electric energy is calculated by the integral calculation of power and time. All the control and operation are completed by DSP, and the adaptive measurement of electric energy is realized. The neural network weights are obtained by using gradient Newton combined learning method.

\section{Experimental results}

Table 1 Comparison of experimental results based on CRDA

\begin{tabular}{|c|c|c|c|c|c|c|c|c|c|c|c|}
\hline \multirow[t]{2}{*}{$\begin{array}{l}\text { Error } \\
\text { preci } \\
\text { sion }\end{array}$} & \multirow{2}{*}{$\begin{array}{l}\text { Maximu } \\
\mathrm{m} \\
\text { number } \\
\text { of } \\
\text { learning } \\
\text { steps }\end{array}$} & \multicolumn{2}{|c|}{$\begin{array}{l}\text { The steepest } \\
\text { descent gradient } \\
\text { algorithm }\end{array}$} & \multicolumn{4}{|c|}{ The ordinary gradient Newton method } & \multicolumn{4}{|c|}{ CRDA method } \\
\hline & & $\begin{array}{l}\text { Number } \\
\text { of } \\
\text { learning } \\
\text { steps }\end{array}$ & $\begin{array}{l}\text { Conve } \\
\text { rgence } \\
\text { ratio }\end{array}$ & $\begin{array}{l}\text { Total } \\
\text { learning } \\
\text { steps }\end{array}$ & $\begin{array}{l}\text { Conver } \\
\text { gence } \\
\text { ratio }\end{array}$ & $\begin{array}{l}\text { The } \\
\text { number } \\
\text { of step } \\
\text { gradient }\end{array}$ & $\begin{array}{l}\text { The } \\
\text { number } \\
\text { of step } \\
\text { Newton }\end{array}$ & $\begin{array}{l}\text { Total } \\
\text { learni } \\
\text { ng } \\
\text { steps }\end{array}$ & $\begin{array}{l}\text { Conve } \\
\text { rgenc } \\
\text { e ratio }\end{array}$ & $\begin{array}{l}\text { The } \\
\text { numbe } \\
\text { r of } \\
\text { step } \\
\text { gradien } \\
\mathrm{t}\end{array}$ & $\begin{array}{l}\text { The } \\
\text { numb } \\
\text { er of } \\
\text { step } \\
\text { Newt } \\
\text { on }\end{array}$ \\
\hline 0.1 & 2000 & 177 & $80 \%$ & 99 & $100 \%$ & 94 & 5 & 96 & $100 \%$ & 88 & 8 \\
\hline 0.01 & 4000 & 499 & $90 \%$ & 297 & $85 \%$ & 291 & 6 & 102 & $92 \%$ & 90 & 12 \\
\hline 0.001 & 8000 & 1221 & $86 \%$ & 460 & $95 \%$ & 448 & 12 & 119 & $98 \%$ & 91 & 28 \\
\hline
\end{tabular}
algorithm with other algorithms smaller the epsilon is, the more the gradient method is used to iterate, and the higher the accuracy of judging the gradient iteration failure, the longer the computation time of the machine.Thus, by using the CRDA method, the point at the end of the gradient iteration method falls into the convergent domain of the Newton iterative method to be used.The effective combination of gradient iteration method and Newton iteration method is achieved.

$W(k+1)=W(k)-\left[\lambda_{k} a_{k} \nabla E(w(k))+\left(1-\lambda_{k}\right)\left[F^{\prime}(W(k))\right]^{-1} F(W(k))\right] \ldots . .(8)$

\section{DSP technology is adopted to realize the real-time fast measurement of electric energy}

Digital signal processor DSP (Digital Signal Processing) is particularly suitable for digital signal processing operations, the main feature is that real-time and rapid implementation of a variety of digital signal processing algorithms. DSP devices work well in common convolution, filtering, FFT and matrix operations. The DSP device produced by the American Simulated company ADSP-21060 is designed for high performance multiprocessing applications such as medical imaging, 3D graphics acceleration, radar, electronic signal processing, etc.It represents the best performing 32 bit DSP chip at present.ADSP-21060 is a high performance floating-point DSP chip, the super Harvard architecture, with four independent bus (two data bus, a program bus
Table . 2 Environmental impact experiment of power measurement

\begin{tabular}{|l|l|l|l|l|l|}
\hline error \environmental & $\begin{array}{l}\text { Temperature } \\
\text { change 40 }\end{array}$ & $\begin{array}{l}\text { Moisture } \\
\text { change } \\
40 \%\end{array}$ & $\begin{array}{l}\text { Voltage change } \\
80 \%\end{array}$ & $\begin{array}{l}\text { Current } \\
100 \%\end{array} \quad$ change & $\begin{array}{l}\text { External magnetic } \\
\text { field change 30\% }\end{array}$ \\
\hline $\begin{array}{l}\text { Adaptive power meter error } \\
\text { change }\end{array}$ & 0.02 & 0.02 & 0.01 & 0.02 & 0.02 \\
\hline $\begin{array}{l}\text { General energy meter error } \\
\text { charge }\end{array}$ & 0.18 & 0.15 & 0.12 & 0.17 & 0.11 \\
\hline
\end{tabular}

The experimental results of different algorithms are shown in table 1.

Repeated studies by Random selection of initial values are completed 100 times, and average results are shown in table 1.We can see from the experimental results of table 1 . The CRDA method to judge the tonsure Newton algorithm significantly reduced the number of learning steps, improve the rate of convergence. The effectiveness of this method is demonstrated.The environmental impact experiment of electric power adaptive measuring instrument based on neural network is carried out. The error of adaptive watt hour meter and general electric energy meter is measured by 0.02 level standard watt hour meter, and the change of the error of two kinds of watt hour meter is compared with the change of environment quantity. The result is shown in table 2 . From the experimental results of Table 2, it can be seen that the error of electric energy measurement in general electric energy meter will cause great additional error when the environment changes.However, the added error of the adaptive energy meter when the environment changes is very small. It basically matches the standard electric energy meter to 
measure the electric energy. The validity of neural network based adaptive measurement method for electric power is verified.

\section{Conclusion}

The adaptive measurement method of power and energy based on neural network can effectively modify its own measurement errors adaptively and in real-time in complex environment. Real time intelligent correction is realized to improve the accuracy of the meter in complex environment. In the neural network learning, the CRDA method is used to reduce the point at the end of the gradient iteration method into the convergent domain of the Newton iterative method to be used, and the gradient iteration method and the Newton iteration method are combined effectively. The adaptive electric energy measurement method will effectively change the traditional principle and calibration method of electric energy measurement, and improve the accuracy, range of application and environmental impact resistance of the meter. This method is of great significance.

\section{Reference}

1. Sulan .Xia , Li.Zhao, Research of multi-sensor information fusion based on BP neural networks [J]computer measurement \& control . 23(5),18231826(2015).

2. Xian $z$.W , Application of fuzzy adaptive backpropagation neural networks in thermal conductivity gas analyzer [J]Neural computing.7(3),579583(2010).

3. Zhangyang. L, Research on multi-sensor date fusion based on improved bp algorithim.[J]Journal of Southeast University(Natural Science Edition) .38(1),258-261(2008).

4. Napolitano. M R, Windon. D A, Casanova J L, et al. Kalman filters and neural-network schemes for sensor validation in flight control systems[J]. IEEE Transactions on Control Systems Technology, 6(5),596-611(1998).

5. Chen.C.L.,Liu'.J.Wen.G.X. Fuzzy neural network based adaptive control For a class of uncertain nolinear stochastic system [J].IEEE Transactions on Cybernetics, 2014,44(5):583-593.

6. Fu Yue,Chai Tianyou, Nonlinear multivariable adaptive control using multiple models and neural networks[J],.Automatica, 2007(43):101-111.

7. Narendira K.S.,Parthasarathy K.,Identification and control of dynamical systems using neural networks, [J].Neural Networks 、 IEEE Transactions on, 1(1),1990,4-27.

8. Rovithakis G.A.,Christodoulou M.A.,Direct adaptive regulation of unknown nonlinear dynamical systems via dynamic neural networks [J].IEEE Transactions on Systems,Man and Cybernetics,25(^n),1995,1578-1594.

9. A.S.Poznyak and E.N.Sanchez,Nonlinear system approximation by neural networks:error stability analysis[J].International Journal of Intelligent Intelligent Automation and Soft Computing, 1995,247-258.
10. A.S.Poznyak,Learning for Dynamic Neural Networks [C].In 10th Yale Workshop.Adaptive Learning Systems, 1998,38-47.

11. A.S.Poznyak,W.Yu,E.N.Sanchez, and J.R Perez,Stability analysis of dynamic neural control[J].Expert System Application, 14(1),1998,227-236.

12. A.RovMiakis and M.A.Christodoulou,Adaptive control of unknown plants using dynamical neural networks[J].IEEE Transaction on Systems,Man and Cybernetics, 1994,(24):400-412.

13. Rovithakis G.A.,Christodoulou M.A.A robust direct adaptive regulation Architecture using dynamic neural network models[C]IEEE Intemational Conference on Systems,Man, and

Cybernetics, 1994.Humans,InformationandTechnology.199 4,(2):1110-115.

14. LiangN.Y.,HuanG.B.,Saratchandran P,et al.A fast and accurate online sequential learning algorithm for feedforward networks[J].Neural Networks,IEEE Transactions on,2006,17(6):1411-1423.

15.Huang G.B.,Wang D.H.,Lan Y.Extreme leaming machines:a survey.International Journal of Machine Learning and Cybemetics[J],2011,2(2):107-122.

16.Huang G.B.,Zhu Q.Y.,Siew C.K.Extreme learning machine:a new Learning scheme of feedforward neural networks[C].Neural etworks,IEEE Intenational Joint Conference on,2004,(2):985-990.

17.Liu G.R,Kadirkamanathan V.Predicti vecontrol for nonlinear systems useing neural networks[J].Intemational Journal of control,1998,71(6):1119-1132.

Acknowledgments: This work was supported by the found of wuyi university (grant 2013-7-112).

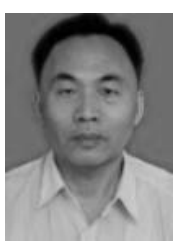

Zhufeng Li . 1965. men . Senior laboratory technician. Master. Mainly engaging in physical experiment, electronic measuring techonlogy and neural net research . Undertake 2 scientific and technological projects in Jiangmen city, 4 research projects funded by Wuyi University. More than 30 papers were published.

Communicate address: 207\# College of Application Physics and material,WuYi Univ. jiangmeng Guangdong China . Zip code: 529020,Telephone: 0750-3299401(o) Email: zhufenglee@126.com

Yang Li. 1991. men .born in harbin china . In 2016, the Bachelor's degree has been awarded from college of life science, state university of Minnesota United States . Studying and internship in state university of Minnesota United States now . Mainly engaging in life science, Genetic engineering, physics, chemical and light application research .In 2017, 1 invention patents were awarded from People's Republic of China, ranking fourth, The patent number is "ZL 201310695565.1". 3 papers were published. 